\title{
Perfil dos óbitos por asfixia perinatal em maternidade pública do sul do Brasil
}

Profile of deaths from perinatal asphyxia in public maternity in southern Brazil

Perfil de muertes por asfixia perinatal en maternidad pública en el sur de Brasil Julia Simões PABIS ${ }^{1}$

Maria Augusta Baptista GUIMBALA ${ }^{1}$

Caroline Louise MACHADO ${ }^{1}$

Patricia Zanotelli CAGLIARI ${ }^{1}$

Milena Luisa SCHULZE ${ }^{1}$

Cristiane Cover SBEGHEN ${ }^{2}$

Maria Volpato KROPIWIEC ${ }^{3}$

Francisco Cesar PABIS ${ }^{1}$

${ }^{1}$ Universidade da Região de Joinville, UNIVILLE, 89249-710 Joinville - SC, Brasil

${ }^{2}$ Maternidade Darcy Vargas, 89202-190 Joinville - SC, Brasil

${ }^{3}$ Secretaria da Saúde - SES, 89221-005 Joinville - SC, Brasil

\section{Resumo}

Introdução: A asfixia perinatal é uma causa importante de mortalidade no período neonatal. Objetivo: O objetivo deste estudo é analisar as características clínico-epidemiológicas dos óbitos ocorridos por asfixia perinatal e comparar com outras causas de óbito no mesmo período. Material e método: Foi realizado um estudo transversal onde foram verificados todos os óbitos ocorridos no período neonatal nos anos de 2009 a 2015 em uma maternidade publica do sul do Brasil. Resultados: Dos 92 casos de óbito $80 \%$ eram prematuros e a principal causa de morte foi Insuficiência respiratória (29\%). Por asfixia perinatal foram 18 casos (19,5\%). Comparando o grupo cuja causa morte foi asfixia com o grupo que morreu por outras causas encontramos diferença no Apgar $(p<0,001)$ e ausência de prematuros extremos $(p<0,05)$. Não houve diferença entre idade no óbito, consultas de pré-natal, idade gestacional, tipo de parto, presença de malformações, uso de oxigênio e peso ao nascer. Conclusão: Portanto observou-se que a morte por asfixia perinatal ocorre na primeira semana de vida, não são prematuros extremos e tem um bom peso ao nascer e quando comparado ao outro grupo o índice de Apgar e ausência de prematuridade extrema mostraram correlação com a morte por asfixia.

Descritores: Asfixia; Assistência Perinatal; Morte Perinatal; Maternidades; Asfixia Neonatal.

\section{Abstract}

Introduction: Perinatal asphyxia is an important cause of mortality in the neonatal period. Objective: The objective of this study is to analyze the clinical and epidemiological characteristics of perinatal asphyxia and to compare it with other causes of death in the same period. Material and method: A cross-sectional study was conducted in which all the deaths occurred in the neonatal period in the years 2009 to 2015 were analyzed. Results: Of the 92 cases of death, $80 \%$ were premature and the main cause of death was respiratory failure (29\%). There were 18 cases of perinatal asphyxia (19.5\%). Comparing the group whose cause death was asphyxia with the group that died from other causes we found difference in Apgar $(p<0.001)$ and absence of extreme preterm infants $(p<0.05)$. There was no difference between age at death, prenatal consultations, gestational age, type of delivery, presence of malformations, use of oxygen and birth weight. Conclusion: Therefore it was observed that death due to perinatal asphyxia occurs in the first week of life, does not occur in extreme prematures and have a good birth weight and when compared to the other group the Apgar score and absence of extreme prematurity showed a correlation with death by asphyxia . Descriptors: Asphyxia; Perinatal Care; Perinatal Death; Hospitals, Maternity; Asphyxia Neonatorum.

\section{Resumen}

Introducción: La asfixia perinatal es una causa importante de mortalidad en el período neonatal. Objetivo: El objetivo de este estudio es analizar las características clínico-epidemiológicas de las muertes por asfixia perinatal y compararlas con otras causas de muerte en el mismo período. Material y método: Se realizó un estudio transversal en el que se verificaron todas las muertes ocurridas en el período neonatal en los años 2009 a 2015 en una maternidad pública del sur de Brasil. Resultados: De los 92 casos de muerte, el $80 \%$ fueron prematuros y la principal causa de muerte fue la insuficiencia respiratoria (29\%). Hubo 18 casos de asfixia perinatal (19,5\%). Al comparar el grupo cuya causa de muerte fue la asfixia con el grupo que murió por otras causas, encontramos una diferencia en Apgar $(p<0,001)$ y ausencia de lactantes extremadamente prematuros $(p<0,05)$. No hubo diferencia entre la edad de fallecimiento, las consultas prenatales, la edad gestacional, el tipo de parto, la presencia de malformaciones, el uso de oxígeno y el peso al nacer.Conclusión: Por lo tanto, se observó que la muerte por asfixia perinatal ocurre en la primera semana de vida, no son extremadamente prematuras y tienen un buen peso al nacer y en comparación con el otro grupo, el puntaje de Apgar y la ausencia de prematuridad extrema mostraron una correlación con la muerte por asfixia.

Descriptores: Asfixia; Atención Perinatal; Muerte Perinatal; Maternidades; Asfixia Neonatal.

INTRODUÇÃO

A asfixia perinatal é causada por uma falta de oxigênio ou má perfusão de vários órgãos levando a alterações bioquímicas e/ou funcionais ${ }^{1}$. A prevalência desta varia na literatura de três a seis por mil nascidos vivos, entretanto a Organização Mundial da Saúde estimou em 1995 que dos 130 milhões de nascimentos mundiais por ano, 4 milhões apresentariam asfixia perinatal ${ }^{2,3}$. Por isso, sendo a asfixia perinatal uma das principais causas de morte evitável do período neonatal e por avaliar a qualidade da assistência ao parto e ao recém-nascido, o objetivo deste trabalho é analisar as características epidemiológicas, e clinicas dos óbitos ocorridos por asfixia perinatal e comparar com as outras causas de óbito ocorridos no mesmo período em uma maternidade pública do sul do Brasil.

MATERIAL E MÉTODO

Estudo transversal observacional retrospectivo de 01 de janeiro de 2009 a 31 de dezembro de 2015, onde foram analisados todos os óbitos ocorridos no período neonatal (até 28 dias de vida) na maternidade Darcy Vargas - Joinville - SC. A Maternidade Darcy 
Vargas, pertence à Secretaria Estadual de Saúde, atua na área de saúde materno-infantil e dispõe de 138 leitos, sendo 96 obstétricos e 42 neonatais. É um "Hospital Amigo da Criança" e centro de referência terciário na região para a assistência do binômio mãe-filho. E referência estadual em Banco de Leite Humano e Método Canguru, ao mesmo tempo em que é a Maternidade de referência em gestação de alto risco para a região Norte e Nordeste de Santa Catarina.

O número de óbitos foi obtido por meio de registro já existentes na Secretária de saúde do Município de Joinville e também foram coletados os seguintes dados em prontuários eletrônicos: idade no óbito, sexo, número de consultas do pré-natal, tipo de parto, idade gestacional, APGAR, presença de malformação congênita, uso de oxigênio, tipo de parto, peso ao nascer e causa do óbito. Foram incluídos todos os óbitos ocorridos no período estudado e excluídos os óbitos sem causa e que não apresentavam o APGAR. O critério utilizado para a classificação da asfixia perinatal foi o de Buonocuore, o qual utiliza os seguintes parâmetros: pH do cordão < 7,2, APGAR do 5은 minuto de vida entre 4 e 6 , FiO2 $\geq 0,40$ para saturar $86 \%{ }^{4}$.

Inicialmente todas as variáveis foram analisadas descritivamente. Para as variáveis quantitativas esta análise foi feita através do cálculo de médias e desvios-padrão. Para as variáveis qualitativas foram calculadas as frequências absolutas e relativas.

Para a análise da hipótese de igualdade entre a média dos dois grupos foi utilizado o teste $\mathrm{t}$ de Student, quando a suposição de normalidade for rejeitada foi utilizado o teste não paramétrico de Mann-Whitney.

Para se testar a homogeneidade dos grupos em relação às proporções foi utilizado o teste qui-quadrado ou o teste exato de Fisher (quando ocorreram frequências esperadas abaixo de 5). O nível de significância utilizado para os testes foi de $5 \%$. Os dados foram analisados pelo programa estatístico SPSS17.

A pesquisa foi conduzida de acordo com as regulamentações da Resolução 466 de 2012 do Conselho Nacional de Saúde, e foi realizada após autorização da Comissão de Ética do Hospital Regional Hans Dieter Schimidt sob o número 1902551.

Foram excluídos os casos com prontuários que não contenham a data de nascimento, data do óbito, idade, escolaridade, número de consultas do pré-natal, comorbidades prévias, tipo de parto, idade gestacional ao nascimento, presença de pediatra na sala de parto, diagnósticos iniciais, considerações e outros diagnósticos importantes.

RESULTADOS

Dos 38440 nascimentos ocorridos na maternidade de 2009 a 2015 ocorreram 130 óbitos, destes 92 casos foram incluídos neste estudo. Nesta amostra $80 \%$ eram prematuros e a principal causa de morte foi Insuficiência respiratória (29\%). Por asfixia perinatal foram 18 casos $(19,5 \%)$. Os casos de Asfixia apresentavam o seguinte perfil: a idade do óbito foi de $(4+-5,8)$ dias, a idade gestacional de $(32+-$ $6,4)$ semanas, Apgar do primeiro minuto em torno de 2 e do quinto minuto de 4 , o peso ao nascer de 2067gramas, $50 \%$ tiveram parto normal e $50 \%$ cesariana, $89 \%$ não tinham malformação associada, metade era do sexo masculino, todos necessitaram do uso de oxigênio e $66 \%$ da amostra eram prematuros (Tabela 1).

Tabela 1. Perfil dos óbitos

\begin{tabular}{lccc}
\hline PERFIL DOS ÓBITOS & & & \\
\hline & ASFIXIA & OUTRAS CAUSAS & P \\
\hline Idade no Óbito (Dias) & 4 & 5 & 0,5 \\
\hline Idade Gestacional (Semanas) & 32 & 29 & 0,2 \\
\hline APGAR 1 ${ }^{\circ}$ minuto & 2 & 4 & 0,001 \\
\hline APGAR 5 5 minuto & 4 & 6 & 0,001 \\
\hline Peso Ao Nascer (Gramas) & $2067+-1101$ & $1492+-959$ & 0,07 \\
\hline Sexo & MASC 50 $\%$ & MASC 54\% & 0,5 \\
& FEM 50\% & FEM 46\% & \\
\hline Uso De Oxigênio & $100 \%$ & $87 \%$ & 0,1 \\
\hline Mal Formação Associada & $11 \%$ & $27 \%$ & 0,1 \\
\hline $\begin{array}{l}\text { Número De Consultas Do Pré- } \\
\text { Natal }\end{array}$ & $6+-4$ & $5+-3,7$ & 0,1 \\
\hline Prematuridade & $60 \%$ & $86 \%$ & 0,05 \\
\hline
\end{tabular}

A principal causa de asfixia foi aspiração de mecônio, as outras causas estão demonstradas na Tabela 2. Comparando o grupo cuja causa morte foi asfixia com o grupo que morreu por outras causas encontramos diferença no Apgar do primeiro minuto $(p<0,001)$, Apgar do quinto minuto $(p<0,01)$, e ausência de prematuros extremos $(p<0,05)$. Não houve diferença entre idade no óbito, número de consultas de pré-natal, idade gestacional, tipo de parto, presença de malformações, uso de oxigênio e peso ao nascer. Dados demonstrados na Tabela 3.

Tabela 2. Causas associadas ao óbito por asfixia

\begin{tabular}{lll}
\hline CAUSA & N & $\mathbf{\%}$ \\
\hline Pneumotórax/Hemorragia Pulmonar & $\mathbf{1}$ & 5,5 \\
\hline Sofrimento Fetal & 1 & 5,5 \\
\hline Hipertensão Pulmonar & 2 & $\mathbf{1 1 , 1}$ \\
\hline Alteração No Cordão Umbilical & 3 & $\mathbf{1 6 , 6}$ \\
\hline Aspiração De Mecônio & 6 & 33,3 \\
\hline Alteração Na Placenta Com Hemorragia & 3 & 16,6 \\
\hline Toco Traumatismo & 1 & 5,5 \\
\hline Toxoplasmose/Hipoglicemia & 1 & 5,5 \\
\hline
\end{tabular}

Tabela 3. Comparação entre asfixia e outras causas de óbito

\begin{tabular}{cccc}
\hline & ASFIXIA & OUTRAS & P \\
\hline APGAR 1 MIN & 2 & 3 & $\mathbf{0 , 0 0 1}$ \\
\hline APGAR 5 MIN & 3 & 5 & 0,001 \\
\hline Idade no óbito & 4 & 4,92 & 0,533 \\
\hline Idade gestacional & 31 & 30 & 0,222 \\
\hline Parto normal & $50 \%$ & $54,9 \%$ & 0,64 \\
\hline Mal formação & $11,1 \%$ & $24,2 \%$ & 0,151 \\
\hline Uso de o2 & $100 \%$ & $90,1 \%$ & 0,119 \\
\hline Peso ano nascer & 20679 & $1593 \mathrm{~g}$ & $\mathbf{0 , 0 7 4}$ \\
\hline Número de consultas do pré-natal & 6 & 5 & $\mathbf{0 , 1}$ \\
\hline
\end{tabular}


DISCUSSÃO

A redução da mortalidade de crianças abaixo de 5 anos é uma meta mundial. No Brasil, onde ocorrem 3 milhões de nascimentos por ano, não é diferente, tanto que é um dos países que aderiu as metas do milênio que foram estabelecidas pela Organização das Nações Unidas(ONU) em 2000, com apoio de 191 nações. O estudo da mortalidade infantil colabora para semalcançar estes objetivos.

Dentre essas mortes, há predomínio das mortes neonatais, principalmente por três causas: prematuridade, infecções neonatais e asfixia perinatal ${ }^{5}$. De acordo com o estudo de Almeida et al. $^{6}$ ocorreram 27.800 mortes neonatais precoces associadas à asfixia entre 2005 e 2010 no Brasil, contribuindo com 40\% de todas as mortes de recém-nascidos de baixo risco. A última causa citada é uma das medidas da qualidade da assistência prestada no período perinatal que, se diagnosticada e tratada precocemente, torna-se altamente evitável. Para que isso ocorra são necessários planejamentos de políticas públicas, e, estas, para serem feitas, necessitam do conhecimento do perfil epidemiológico desses óbitos ${ }^{7}$.

A prematuridade vem sendo apontada como a principal contribuinte da mortalidade neonatal ${ }^{\text {. }}$. Nos nossos resultados, houve predomínio de prematuros, representando $80 \%$ da amostra. Estudos demonstram que os recém-nascidos prematuros apresentam maior susceptibilidade a eventos adversos. As consequências da prematuridade refletem tanto a imaturidade de seus órgão e sistemas quanto as intensivas intervenções necessárias à sua sobrevivência ${ }^{8-10}$. Um dos eventos mais importantes a que o recém-nascido está sujeito é a hipóxia. Esta pode ser definida como sendo uma inadequada oxigenação em nível celular, na maioria das vezes representada pela presença de cianose. Os critérios utilizados para se determinar hipóxia, além da presença ou ausência de cianose, são valores de Apgar igual ou menor que 6 , necessidade de ressuscitação contínua, acidose profunda ( $\mathrm{pH}$ menor que 7 ou déficit de base de $16 \mathrm{mmol} / \mathrm{L}$ ou mais) e evidências de encefalopatia hipóxico-isquêmica na avaliação neurológica (letargia, torpor, coma, hipotonia ou reflexos anormais) $)^{11-14}$.

As sequelas de um evento hipoxêmico dependem de sua intensidade e de sua duração. Em alguns casos, o cérebro pode ser o único orgão acometido. Em uma série com 57 crianças, a encefalopatia hipóxico-isquêmica isolada ocorreu em 14 delas (24,5\%). Em outro estudo retrospectivo com 130 neonatos com hipóxia, os outros sistemas que apresentaram disfunção foram o renal (70\%), o cardiovascular $(62 \%)$, o pulmonar $(86 \%)$ e o hepático $(85 \%)^{8}$. A importância da monitorização precoce, de medidas que minimizem a hipóxia e do estabelecimento de critérios clínicos e laboratoriais que norteiem estes cuidados são de suma importância para a sobrevida destes pacientes.

Quantificar a hipóxia em prematuros é uma tarefa difícil. $O$ índice de Apgar é um dos parâmetros mais utilizados e pode estar baixo sem que o recém-nascido prematuro tenha apresentado acidose fetal.

Para traçar esse perfil é primeiramente necessário conhecer as definições e diferenças de hipóxia e asfixia. A hipóxia é uma inadequada oxigenação a nível celular, representada pela presença de cianose (coloração azulada da pele, língua e mucosas). Portanto, é uma deficiência de oxigênio no sangue arterial ${ }^{8}$. Já o termo asfixia deverá ser utilizado, de acordo com a Academia Americana de Pediatria, quando houver acidemia metabólica ou mista profunda $(\mathrm{pH}<7)$ em sangue arterial em cordão umbilical, escore de APGAR de 0-3 por mais de 5 minutos, manifestações neurológicas neonatais (convulsão ou hipotonia), disfunção orgânica multissistêmica. Contudo, há outro conceito utilizado, de Buonocuore, o qual utiliza os seguintes parâmetros: $\mathrm{pH}$ do cordão $<7,2$, APGAR do $5^{\circ}$ minuto de vida entre 4 e $6, \mathrm{FiO} 2$ $>=0,40$ para saturar $86 \%{ }^{4}$.

A prevalência da asfixia perinatal no total de óbitos na Maternidade Darcy Vargas foi de $18 \%$, sendo que no estudo de Almeida et al. ${ }^{6}$ que avaliou todas as mortes neonatais precoces ocorridos no Brasil entre 2005 e 2010, a asfixia perinatal por aspiração de mecônio representou $30 \%$ de todas as mortes por asfixia6. Já no estudo de Nascimento et al. ${ }^{15}$ foi de 2,15 e no de Cruz e $\mathrm{Ceccon}^{4}$ foi de 0,32. Essa diferença significativa dos dados apontados pode decorrer do fato de ter sido utilizado o método de Buonocore para a classificação das asfixias deste estudo. Entre as causas da asfixia o sofrimento fetal ocorreu em $5,5 \%$ neste estudo e em $23,3 \%$ no de Cruz e Ceccon ${ }^{4}$. Quanto à aspiração de mecônio foi encontrado 33,3\% nesse estudo e $10 \%$ no de $\mathrm{Cruz}^{4}$, demonstrando uma diferença significativa e que deve ser avaliada. Já a alteração no cordão umbilical ocorreu em $16,6 \%$ e no estudo de Cruz e Ceccon $^{4}$ em $3,3 \%$, as alterações na placenta foram observadas em $16,6 \%$ e em Nascimento et al. $^{15}$ em $14,8 \%$. E a prematuridade demonstrou-se prevalente em todos os estudos. Avaliamos nesta pesquisa outros itens, 
já apresentados nos resultados, que não encontramos em estudos nacionais, mas que são preditores para a avaliação do perfil do óbito e dentre essas é possível perceber as causas evitáveis a fim da elaboração de políticas públicas efetivas e que melhorem a qualidade da assistência ao parto e ao recém-nascido.

Além disso, é notada no Brasil, uma diferença significativa entre as regiões, sendo que a taxa de mortes por asfixia perinatal é duas vezes maior nas regiões norte e nordeste se comparado à região sul, local deste estudo. Além disso, a implantação da rede cegonha pelo governo federal, em 2011, e o credenciamento da Maternidade do presente estudo, também pode ter influenciado na diferença de prevalência da asfixia perinatal por melhorar a qualidade e ampliar o acesso ao cuidado perinatal ${ }^{6}$.

CONCLUSÃO

Observou-se que a morte por asfixia perinatal ocorre na primeira semana de vida, não são prematuros extremos e tem um bom peso ao nascer, e quando se comparando ao outro grupo, o índice de Apgar e ausência de prematuridade extrema mostraram correlação com a morte por Asfixia. Além disso, não houve diferença entre idade no óbito, número de consultas de pré-natal, idade gestacional, tipo de parto, presença de malformações, uso de oxigênio e peso ao nascer.

Em nosso país há carência de informações e estudos nessa faixa etária e, por isso, espera-se que esse tipo de estudo ajude a identificar problemas e planejar soluções a fim de aprimorar o cuidado ao binômio mãe-filho' .

\section{REFERÊNCIAS}

1. Levene ML, Kornberg J, Williams THC. The incidence and severity of postasphyxial encephalopathy in full-term infants. Early Hum Dev. 1985;11:21-6.

2. World Health Organization. Neonatal and perinatal mortality: country, regional and global estimates. Geneva: WHO; 2006. 5.

3. Volpe JJ. Neurology of the newbon. 4th ed. Philadelphia: W.B. Saunders Company; 2000. p.217-394.

4. Cruz ACS, Ceccon MEJ. Prevalência de asfixia perinatal e encefalopatia hipóxico-isquêmica em recém-nascidos de termo considerando dois critérios diagnósticos. Rev bras crescimento desenvolv hum. 20(2):302-16.

5. Daripa M, Caldas HM, Flores LP, Waldvogel BC, Guinsburg R, de Almeida MF. Perinatal asphyxia associated with early neonatal mortality: populational study of avoidable deaths. Rev Paul Pediatr. 2013;31(1):37-45.

6. Almeida MFB, Kawakami MD, Moreira LMO, Santos RMV, Anchieta LM, Guinsburg R. Early neonatal deaths associated with perinatal asphyxia in infants $\geq 2500 \mathrm{~g}$ in Brazil. J Pediatr. 2017;93(6):576-84.

7. BRASIL. Secretaria de Vigilância. Ministério da Saúde. Manual do Óbito de Vigilância infantil e fetal e do Comitê de prevenção do óbito infantil e fetal. 2009.

8. Lawn JE, Cousens S, Zupan J. Lancet Neonatal Survival Steering Team. 4 million neonatal deaths: When? Where? Why? Lancet. 2005;365(9462):891-900.

9. Cloherty JP, Eichenwald EC, Stark AR. Manual of Neonatal Care. 6. ed, Lippincott Williams \& Wilkins, 2007.

10. McCormick MC, Litt JS, Smith VC, Zupancic JA. Prematurity: an overview and public health implications. Annu Rev Public Health. 2011;32:367-79.

11. Gluckman PD, Wyatt JS, Azzopardi D, Ballard $R$, Edwards $A D$, Ferriero DM, Polin RA, Robertson CM, Thoresen M, Whitelaw A, Gunn AJ. Selective head cooling with mild systemic hypothermia after neonatal encephalopathy: multicentre randomised trial. Lancet. 2005;365(9460):663-70.

12. Wiggglesworth $S$, Singer DB. Textbook of fetal and perinatal pathology. 2. ed. Massachusetts: Blackwell Science; 1998.

13.Zhang L, Xue Q. Prenatal hypoxia causes a sex-dependent increase in heart susceptibility to ischemia and reperfusion injury in adult male offspring: role of protein kinase $\mathrm{C}$ epsilon. $\mathrm{J}$ Pharmacol Exp Ther. 2009;330(2):624-32.

14. Rohan AJ, Golombek SG. Hypoxia in the term newborn - part one: cardiopulmonary physiology and assessment. The American Journal of Maternal Child Nursing. 2009; 34(3):144-52.

15. Nascimento SB, Alves AS, Silva APD, Andrade FA, Souza MCA, Castañeda DFN et al. Prevalência e fatores associados à anóxia perinatal nas maternidades de Aracaju e sua repercussão sobre a mortalidade infantil. II Seminário de Pesquisa FAP-SE. Aracaju, 2004. 


\section{CONFLITO DE INTERESSES}

Os autores declaram não haver conflitos de interesse

\section{AUTOR PARA CORRESPONDÊNCIA}

\section{Milena Luisa Schulze}

Department of Medicine

University of Joinville's Region UNIVILLE

Paulo Malschitzki Street, 10 - Zona Industrial Norte,

89249-710 Joinville - SC, Brazil

E-mail: milenaluisa.sch@gmail.com

Submetido em 01/09/2020

Aceito em 12/02/2021 